\title{
A Successful Kidney Transplant for a Case of LMX1B Gene Mutation with a Positive Family History of Polycystic Kidney Disease
}

\author{
El-Kossi M*, Sherazee T and Halawa A
}

Doncaster and Bassetlaw Teaching Hospitals NHS Foundation Trust Doncaster, South Yorkshire, UK

\begin{abstract}
Nail-Patella syndrome is pleiotropic autosomal dominant disorder characterized by skeletal abnormalities and frequently renal disease. In this case report genetic analysis revealed a pathogenic heterozygous missense mutation of the LMX1B gene as c.[737G>A], p.([Arg246GIn)]. This variant is predicted to cause substitution of a basic arginine residue for a polar uncharged glutamine residue at position 246 . The nucleotide at position 737 and amino acid at position 246 are both highly conserved across species. Although LMX1B mutation causing hereditary focal segmental glomerulosclerosis (FSGS) without any extra-renal involvement has been described as nail patella like renal disease (NPLRD) where there is partial impairment of transcriptional activity with these mutations leading to absence of other features of nail patella syndrome (NPS). Our case report gets interesting as patient had progressed relatively quickly to end stage renal disease. Some of heterozygous gene mutation FSGS cases are expected to recur after kidney transplantation. Our case has received a successful renal transplant from brother which is working well for the last 3 years without any evidence of recurrence. Another interesting feature of our case was the strong family history of unrelated other renal genetic disorder autosomal dominant adult polycystic kidney disease (ADPKD).
\end{abstract}

\section{Keywords: FSGS; LMX1B; NPS; Kidney transplantation}

\section{Introduction}

Steroid resistant nephrotic syndrome (SRNS) is the second cause of end stage renal disease in the first two decades of life in some registries [1]. The list of genetic disorder as a cause of this problem is expanding. Around 39 genes were identified as a cause of this disorder. LMX1B gene mutation is one of these genes in the list [2]. It encodes the Lin11, Isi-1 and Mec-3 (LIM) homeodomain. Protein product of this gene interacts with transcriptional factors that play an important role in glomerular development and morphogenesis in addition to other functions including limb development [3]. The role of LMX1B gene mutation in renal disorders was first recognized as a cause of the NPS [4]. NPS is characterized by autosomal-dominant inheritance with skeletal abnormalities in the majority of the patients. These include dysplastic nails, absent or hypoplastic patellae, elbow dysplasia, iliac horns [5]. Around 40\% of NPS patients develop renal involvement that varies from mild proteinuria to end stage renal disease [6]. Renal limited phenotype of NPS due to LMX1B gene mutation was described by many authors $[2,7,8]$. A condition which is termed NPLRD and was first described by Salcedo (1984) when genetic analysis was lacking at that time. He described a male boy a product of a consanguineous marriage with proteinuria, haematuria and mild renal impairment. $\mathrm{He}$ exhibited same histopathologic features on electron microscopy of NPS without the phenotypic features of limb or nail changes characteristic of this disorder. Several mutations were described of LMX1B gene with the most common mutation encountered in NPLRD is R246Q [2]. We here present a SRNS case with similar mutation in the LMX1B gene which progressed to end stage renal disease requiring renal replacement therapy at the age of 22 years. Progression to ESRD is not universal and it usually develops late [6]. There are few cases with the full NPS phenotype who progressed to end stage renal disease (ESRD) in their early childhood. The gene mutation in our case was de novo. More interestingly she successfully received kidney transplantation from her brother with normal transplant function for the last 3 years without any evidence of proteinuria. Of interest this patient has a strong family history of another unrelated genetic renal disorder ADPKD.

\section{Case History}

A 17-years-old girl referred to renal clinic by ophthalmologist as she was noted to have malignant hypertension $(210 / 115 \mathrm{~mm}$ of $\mathrm{Hg})$ with papilloedema. She had very minimal pedal edema and on routine testing in clinic she was found to have urine PCR of $1180 \mathrm{mg} / \mathrm{mmol}$ and serum albumin of with normal renal function. Kidney biopsy was done which confirmed the diagnosis of primary FSGS. She was initially treated with high dose of steroids to which she failed to respond. She was then treated with combination of steroids and tacrolimus to which she had a partial response when her urine (protein creatinine ratio) PCR improved to an average of $500 \mathrm{mg} / \mathrm{mmol}$ down from $1200 \mathrm{mg} /$ mmol. Tacrolimus was discontinued after 15 months due to side effects and decline in kidney function. She was then given a trial of Mycophenolate Mofetil to which she did not respond either. During this time there was apparently improvement in her eGFR from $45 \mathrm{ml} /$ $\min$ to $60 \mathrm{ml} / \mathrm{min}$, but she continued to have heavy proteinuria. After that she was treated with cyclophosphamide which again proved to be unsuccessful and kidney function continued to deteriorate. At this point her father was willing to donate her a kidney. A genetic analysis was done prior to consider the transplantation option which showed LMX1B gene mutation as the cause of primary FSGS and an answer to why she failed to treatment.

She did not have any other features of Nail-patella syndrome. Her father was tested for same gene which was negative.

*Corresponding author: Dr. Mohsen El-Kossi, Doncaster and Bassetlaw Teaching Hospitals NHS Foundation Trust Doncaster, South Yorkshire, UK, Tel: +44-130236-6666; E-mail: elkossi@gmail.com

Received September 25, 2018; Accepted October 08, 2018; Published October 11, 2018

Citation: El-Kossi M, Sherazee T, Halawa A (2018) A Successful Kidney Transplant for a Case of LMX1B Gene Mutation with a Positive Family History of Polycystic Kidney Disease. J Mol Genet Med 12: 369 doi:10.4172/1747-0862.1000369

Copyright: @ $2018 \mathrm{El}-\mathrm{Kossi} \mathrm{M}$, et al. This is an open-access article distributed under the terms of the Creative Commons Attribution License, which permits unrestricted use, distribution, and reproduction in any medium, provided the original author and source are credited 
Citation: El-Kossi M, Sherazee T, Halawa A (2018) A Successful Kidney Transplant for a Case of LMX1B Gene Mutation with a Positive Family History of Polycystic Kidney Disease. J Mol Genet Med 12: 369 doi:10.4172/1747-0862.1000369

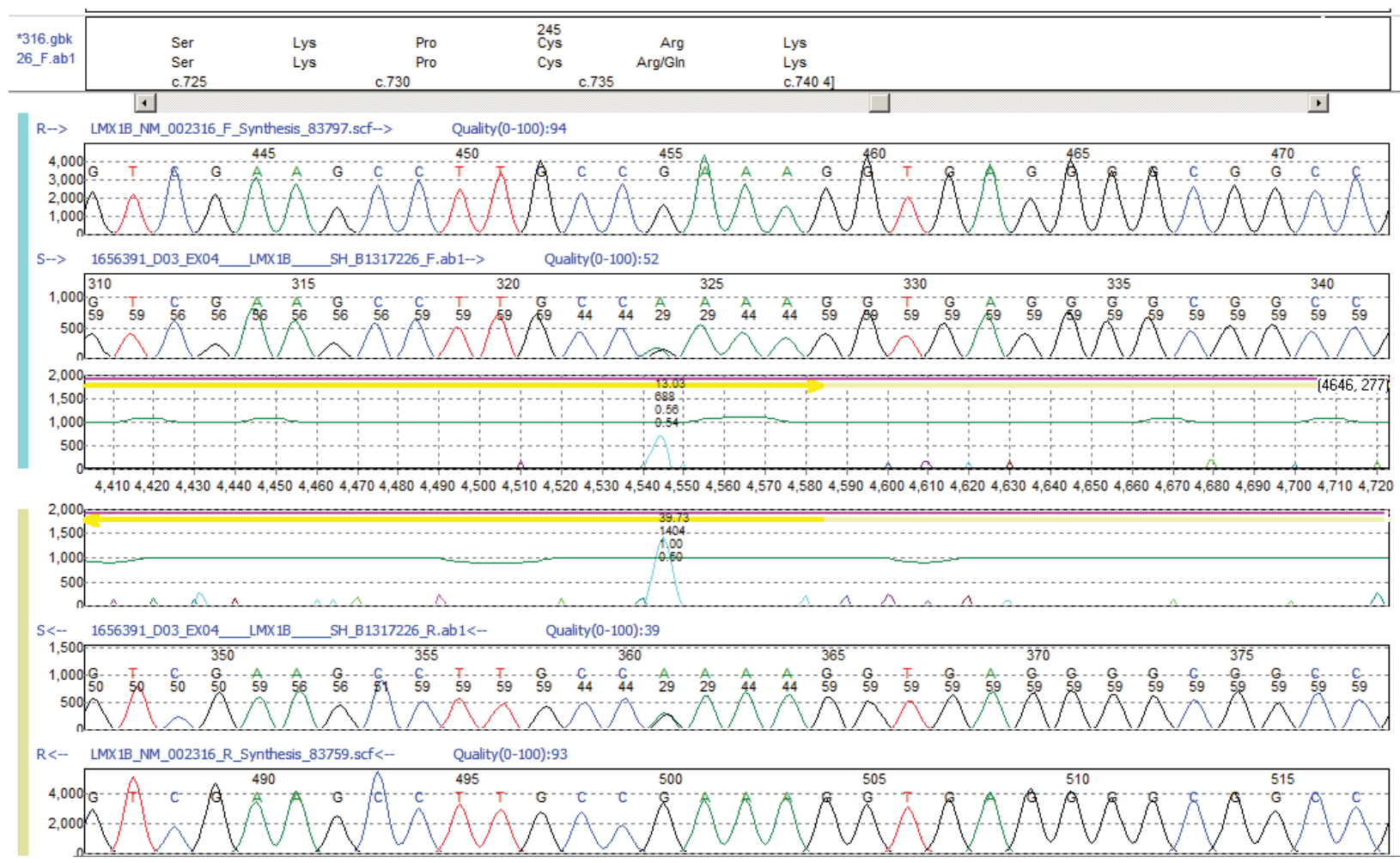

Figure 1: Bidirectional sanger sequencing of exon 4 of the LMX1B gene. 'A' shows the reference sequence (Wild Type). 'B' shows the patient result, at position c.737 the guanine nucleotide is heterozygously replaced with adenine, resulting in a predicted Arginine to Glutamine amino acid change. ' $C$ ' highlights the presence of a difference between the reference sequence and patient sequence.

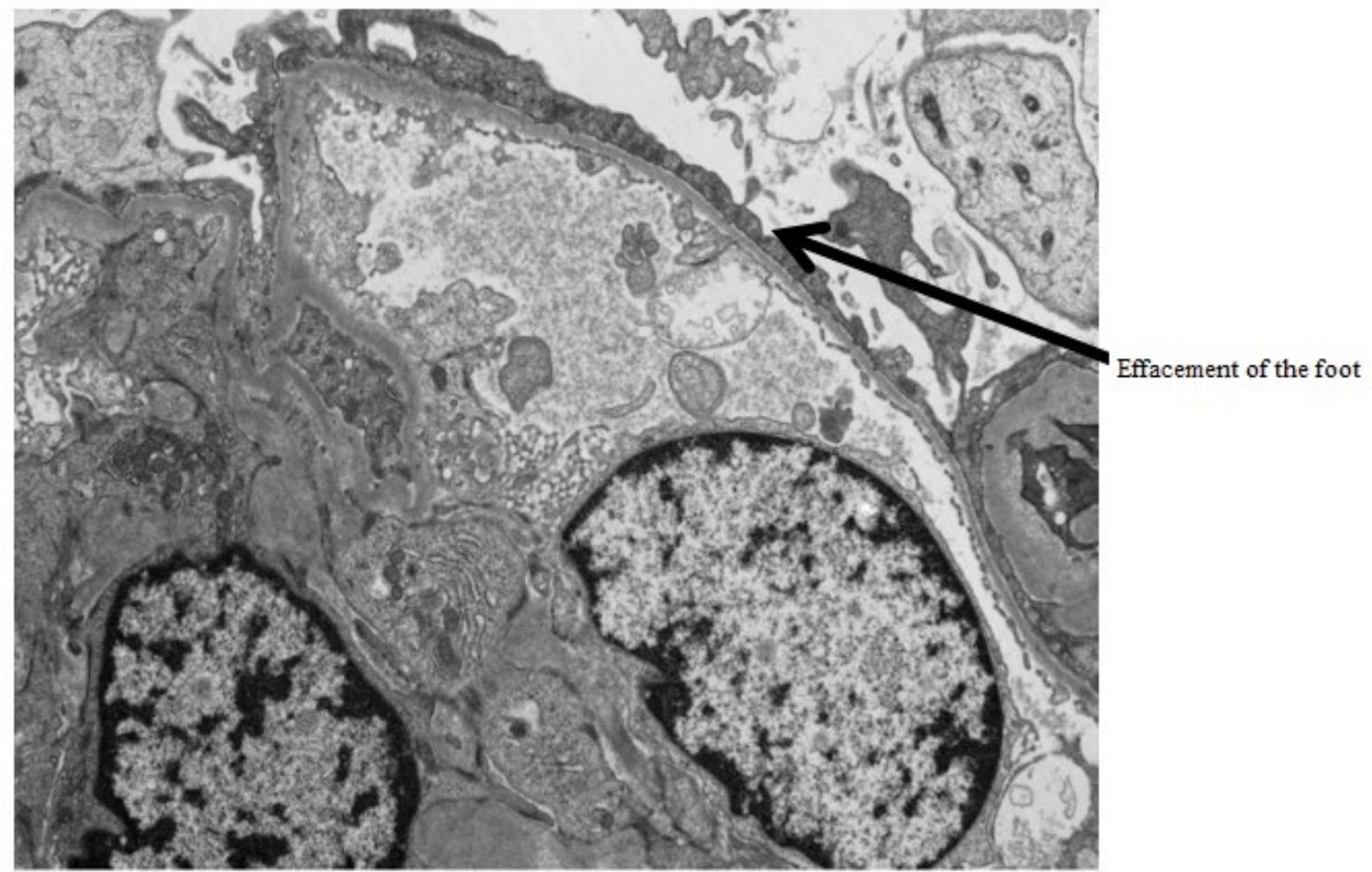

Figure 2: Electron micrograph of renal biopsy showing glomerulus with effacement of the foot process of the podocytes with homogenous thickness glomerular basement membrane (Magnification 2600x). 


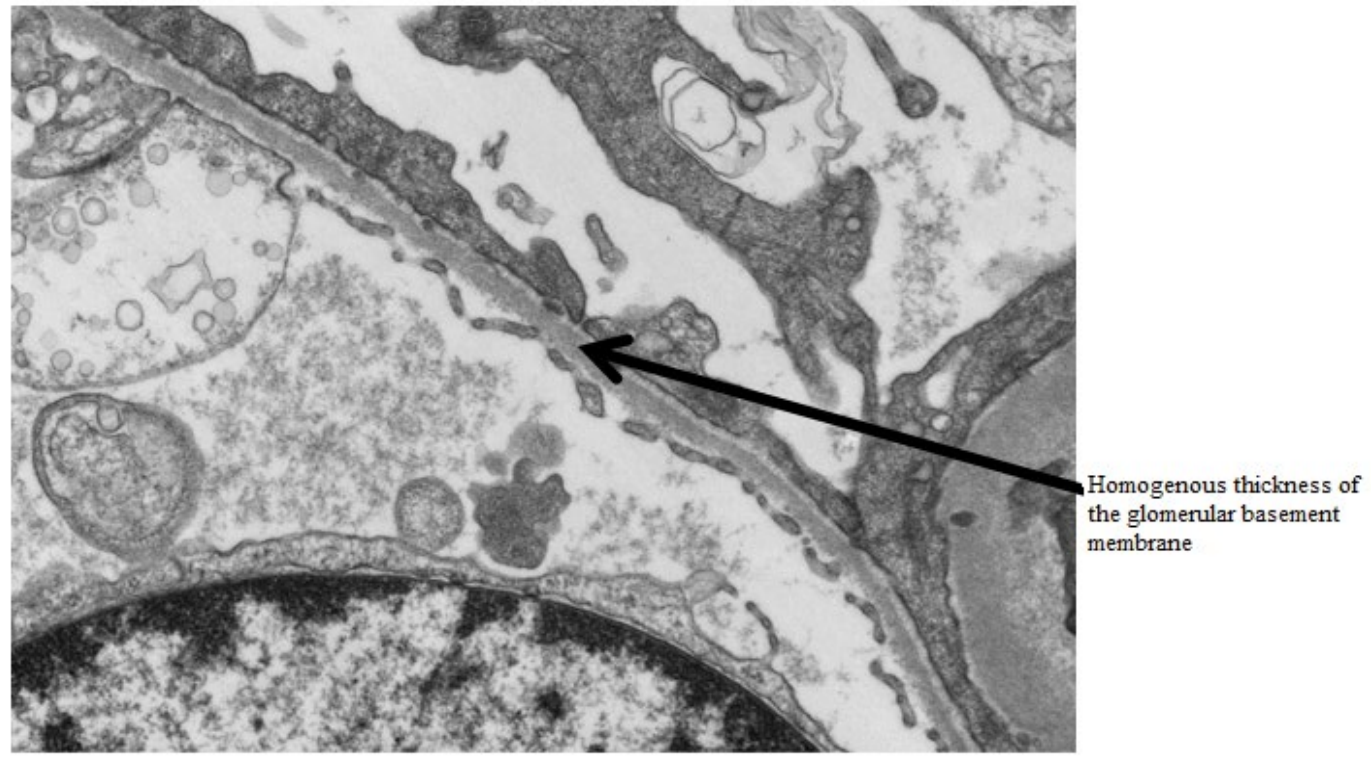

Figure 3: Higher magnification (9600x) electron micrograph of a glomerulus shows the same features of foot process effacement of the podocytes and homogenous thickness of the glomerular basement membrane. There is no evidence of moth-eaten appearance.

Interestingly from her paternal side she had a strong family history of ADPKD. Her paternal grandmother, uncle and aunt all were diagnosed with ADPKD. US of the father's kidneys did not reveal any evidence of ADPKD at age of 40 years. Later she received transplant form her brother who was also negative for LMX1B gene mutation or ADPKD. The transplanted kidney is working very well with normal kidney function for the last three years without any evidence of disease recurrence.

\section{Method}

This patient was tested for 37 genes associated with SRNS using a haloplex target enrichment system and Next Generation Sequencing. Genes screened in the panel included ACTN4, ALG1, ALMS1, APOL1, ARHGAP24, ARHGDIA, CD151, CD2AP, COL4A3, COL4A4, COL4A5, COQ2, COQ6, COQ7, COQ9, CYB11B2, E2F3, INF2, ITGA3, ITGB4, KANK2, LAMB2, LMX1B, MED28, MYH9, MYO1E, NPHS1, NPHS2, PDSS2, PLCe1, PMM2, PTPRO, SCARB2, SMARCAL1, TRPC6, WT1, ZMPSTE24. The result of this sequencing is depicted in Figure 1.

\section{Discussion}

Prognosis of LMX1B-associated nephropathy is very variable. Literature review revealed NPLRD cases with R246Q LMX1B mutation may progress to ESRD around middle age. Konomoto and colleagues (2016) referred to two cases with similar mutation progressed to ESRD at age of 38 and 40 years. Boyer and colleagues (2013) described 5 cases with a similar mutation progressed to ESRD between ages of 36 and 70 years. In the same year (2013), Isojima and colleagues described a 6-year-old Japanese girl with the same LMX1B gene mutation and NPLRD in a mild form. She has had normal kidney function with mild proteinuria and microscopic haematuria. Our case presented with malignant hypertension and nephrotic range proteinuria at the age of 17. She progressed to ESRD by the age of 22 and received successfully live related kidney transplant at the age of 23 . Such huge variability in the natural course of these cases including age and severity of renal involvement at presentation with or without progression to ESRD probably reflect either genetic or other non-genetic factors that could contribute to this wide range of disease spectrum.

Of interest a pair of identical twins with NPS one progressed rapidly to renal failure whilst the other remained only proteinuric [9]. In the interesting Pittsburgh study (2002), a cross sectional review of 25 children and 32 adults with genetic diagnosis of NPS, there was no reported case of ESRD in that cohort. One adult had stage one hypertension, six children and eight adults did have proteinuria [10]. Factors normally known to adversely affect chronic kidney disease progression including blood pressure control, high cholesterol level and heavy proteinuria were treated adequately in this patient with specific measures including statin use, full blockage of angiotensin converting enzyme (ACE) and the use of calcinurin inhibitors (CNI) inhibitor Tacrolimus. These measures reflected on significant improvement of the urine protein creatinine ratio from around 1200 $\mathrm{mg} / \mathrm{mmol}$ to an average of around $400 \mathrm{mg} / \mathrm{mmol}$ with improvement of serum albumin from $22 \mathrm{~g} / \mathrm{l}$ to around $30 \mathrm{~g} / \mathrm{l}$. These changes have particularly taken place when she was using the CNI probably due to the non-immune CNI effects on podocytes [11]. Of relevance, patient adherence to medications was evident from the occasional improvement in proteinuria and the poor tolerance to Tacrolimus due to side effects particularly hair fall. Whilst the patient has a strong family history of ADPKD, US examination of the patient did not reveal any evidence of cystic changes in her kidneys that could contribute to disease progression. The alternative contributing factor to disease progression in her case could be the nature and severity of genetic mutation. Genetic analysis revealed heterozygous pattern with the missense fault at $[737 \mathrm{G}>\mathrm{A}]$ resulting in the same mutation and protein structure (R246Q) previously described in the literature but with different disease course to our case. Lemley (2009) hypothesised an explanation to help explain the wide spectrum of renal involvement in NPS and to justify the discrepancy in the course of CKD progression. He suggested the presence of two distinct pathways. One pathway experienced by the majority of cases of renal involvement in NPS (around 95\%) who shows age related drop of glomerular filtration rate 
at nearly twice that of natural aging process rate. Not all patients in this group develop proteinuria and attributed disease progression to progressive glomerular basement membrane thickening and podocyte dysfunction. On the other hand, there is a minority of cases (around $5 \%$ ) run relatively rapid progressive course with more proteinuria. The latter group probably fits well with our case but without the skeletal deformities of NPS. To explain the discrepancy in the behaviour of both groups, he postulated the presence of another heterozygous mutation or polymorphism in the exons, promoter or enhancer elements of a gene pivotal to podocyte function in addition to LMX1B heterozygous single gene mutation. He referred to possible set of genes that could support his theory including NPHS1, NPHS2, CD2AP and ACTN4, that could be responsible for the rapid rate decline of GFR either directly or indirectly through functions regulated by LMX1B gene. Interestingly, our case was tested for mutations of around 37 potential genes that could be responsible for the steroid resistant status with the above genes were part of this set. There was no evidence of any mutation in any of these genes identified.

Interestingly genetic contribution to the severity of nail disorder in NPS was studied in depth by Dunstin and colleagues (2005) but no similar study was done for cases with renal involvement. There were no unique histological features either in our case to predict the rapid deterioration of kidney function. Histological examination revealed typical picture of FSGS on light microscopy and EM. It showed clear effacement of the podocyte foot process as shown in electron micrographs in Figures 2 and 3 without any evidence of other EM features characteristic of NPS such as moth eaten appearance or irregular thickening of the glomerular basement membrane Of interest, renal ultrastructural changes characteristic of NPS may be found in some patients without clinical evidence of renal involvement and the severity of pathological changes do not correlate with clinical severity or prognosis [12]. Such variability of the spectrum of renal phenotypic presentation in both NPS and NPLRD, with absence of phenotypic skeletal disorders in NPLRD cases, it remains unclear the exact cause of this discrepancy despite of the similar genetic disorder. Of interest to our case there is a strong family history of ADPKD from the parenteral side. Uncle had a successful kidney transplant because of this disorder, grand mum died of ESRD secondary to ADPKD and her aunt developed ESRD because of the same problem. Father was considered negative for this disorder when he was screened as a potential live donor and did not have any cysts on US screening and her brother who was a donor has had a genetic analysis for ADPKD and did not show any evidence of this disorder. Whilst this is an interesting observation we do not think that there is any relationship with the current genetic mutation. The last important point is the successful kidney transplantation from her brother who was negative for the same genetic mutation. After 3 years of the transplantation, the patient still has normal kidney function without any evidence of disease recurrence. This confirms the absence of recurrence of this disorder post kidney transplantation. There are few reports to suggest a potential recurrence in some hereditary forms of FSGS particularly heterozygote cases of SRNS with autosomal recessive inheritance of NPHS2 mutation [13].

\section{Conclusion}

This case extends the finding of LMX1B R246Q mutation as a cause of NPLRD. This mutation may present in adolescent with severe hypertension and nephrotic range proteinuria with minimal peripheral oedema. It can result in ESRD at relatively younger age and risk of disease recurrence post live related kidney transplantation is negligible. It would also be advisable to avoid any of the cytoxic medications in SRNS prior to genetic analysis.

\section{Acknowledgment}

Authors acknowledge the great effort and help of Mrs Laura Yarram-Smith who did the genetic analysis of the case and provided the graph of the gene sequencing.

\section{References}

1. Tasic V, Gucev Z, Polenakovic M (2015) Steroid resistant nephrotic syndrome: Genetic consideration. Pril Makedon Akad Nauk Umet Odd Med Nauki 36: 5-12.

2. Boyer O, Woerner S, Yang F, Oakeley EJ, Linghu B, et al. (2013) LMX1B mutations cause hereditary FSGS without extrarenal involvement. J Am Soc Nephrol 24: 1216-1222.

3. Dai JX, Johnson RL, Ding YQ (2009) Manifold functions of the nail-patella syndrome gene LMX1B in vertebrate development. Dev Growth Differ 51: 241 250 .

4. Dreyer SD, Zhou G, Baldini A, Winterpacht A, Zabel B, et al. (1998) Mutations in LMX1B cause abnormal skeletal patterning and renal dysplasia in nail patella syndrome. Nat Genet 19: 47-50.

5. Sweeney E (2003) Nail patella syndrome: A review of the phenotype aided by developmental biology. J Med Genet 40: 153-162.

6. Bongers EM, Gubler MC, Knoers NV (2002) Nail-patella syndrome: Overview on clinical and molecular findings. Pediatr Nephrol 17: 703-712.

7. Isojima T, Harita Y, Furuyama M, Sugawara N, Ishizuka K, et al. (2014) LMX1B mutation with residual transcriptional activity as a cause of isolated glomerulopathy. Nephrol Dial Transplant 29: 81-88.

8. Konomoto $\mathrm{T}$, Imamura $\mathrm{H}$, Orita M, Tanaka E, Moritake $\mathrm{H}$, et al. (2016) Clinical and histological findings of autosomal dominant renal-limited disease with LMX1B mutation. Nephrol 21: 765-773.

9. Heidet L, Bongers EM, Sich M, Zhang SY, Loirat C, et al. (2003) In vivo expression of putative LMX1B targets in nail-patella syndrome kidneys. Am J Pathol 163: 145-155.

10. Lemley KV (2009) Kidney disease in nail-patella syndrome. Pediatr Nephrol 24: $2345-2354$

11. Wakamatsu A, Fukusumi Y, Hasegawa E, Tomita M, Watanabe T, et al. (2016) Role of calcineurin $(\mathrm{CN})$ in kidney glomerular podocyte: $\mathrm{CN}$ inhibitor ameliorated proteinuria by inhibiting the redistribution of $\mathrm{CN}$ at the slit diaphragm. Physiol Rep 4: e12679.

12. Bennett WM, Musgrave JE, Campbell RA, Elliot D, Cox R, et al. (1973) The nephropathy of the nail-patella syndrome. Clinicopathologic analysis of 11 kindreds. Am J Med 54: 304-319.

13. Weber S, Tönshoff B (2005) Recurrence of focal-segmental glomerulosclerosis in children after renal transplantation: Clinical and genetic aspects. Transplant 80: S128-S134. 